DOI: $10.1002 /(($ please add manuscript number $))$

Article type: Communication

\title{
Direct patterning of organic functional polymers through conventional photolithography and non-invasive cross-link agents.
}

Marco A. Squillaci, Feng Liu, Fan Zhang, Alessandro Aliprandi, Xinliang Feng ${ }^{*}$ and Paolo Samori*

((Optional Dedication))

M. A. Squillaci, Dr. A. Aliprandi, Prof. Dr. P. Samorì

ISIS \& icFRC, University of Strasbourg \& CNRS, 8 Allée Gaspard Monge, 67000

Strasbourg, France.

E-mail: samori@unistra.fr

Dr. F. Liu, Prof. Dr. F. Zhang, Prof. Dr. X. Feng.

School of Chemistry and Chemical Engineering, Shanghai Jiao Tong University, Shanghai 200240, P. R. China

Prof. Dr. X. Feng

Center for Advancing Electronics Dresden (CFAED) \& Department of Chemistry and Food Chemistry, Technische Universität Dresden, Mommsenstraße 4, 01062 Dresden, Germany E-mail: xinliang.feng@tu-dresden.de

Keywords: photoresist, boron-cored chromophore, patterning

Molecules and macromolecules are versatile building blocks whose properties can be tuned via chemical functionalization. Their processing into ordered self-assembled materials on flexible substrates using upscalable methods opens perspectives towards large-area application. ${ }^{[1-2]}$ Albeit the past decades have witnessed a remarkable increase in the performance of organic electronic devices such as thin-film transistors, ${ }^{[3-5]}$ light-emitting diodes, ${ }^{[6]}$ solar cells ${ }^{[7]}$ and sensors, ${ }^{[8]}$ very few are the patterning techniques that are compatible with these materials. ${ }^{[9]}$ Different from their inorganic counterparts, organic materials are unstable (e.g. get swollen) when exposed to the organic solvents typically used for the semiconducting processing. ${ }^{[10]}$ To circumvent this problem, patterning techniques, friendly with organic semiconductors, have been developed. Hitherto, the most promising 
techniques are inkjet printing ${ }^{[11-13]}$ and nano-imprint lithography (NIL). ${ }^{[14-16]}$ Both methods are relatively inexpensive and suitable for large-area patterning, yet they exhibit some disadvantages. In the case of inkjet printing the slow evaporation speed of the solvent leads to a loss of homogeneity due to dewetting phenomena (like the coffee-ring effect), ${ }^{[17]}$ whereas in the case of the NIL it is possible to reach a resolution of tens on nanometers but the use of stamps to transfer the pattern onto technologically relevant supports reduces the versatility and the possibility of producing multilayer structures. ${ }^{[18]}$ The use of blends of functional polymers with cross-link agents represents a novel and facile approach to pattern organic materials with unprecedented versatility. In such a method reported in 2010 by Png et al. the cross-link agents can be activated using a powerful deep-ultraviolet light $(\lambda=254 \mathrm{~nm})$, without interfering with the functional properties of the starting organic semiconductors, to realize high performance multicomponent devices. ${ }^{[19]}$

Here we report on a new scaffold: 5,5,11,11,17,17-hexafluoro-6,12,18-trihexyl$5,6,11,12,17,18$-hexahydro- $4 \mathrm{~b} \lambda^{4}, 6,10 \mathrm{~b} \lambda^{4}, 12,16 \mathrm{~b} \lambda^{4}, 18$-hexaaza- $5 \lambda^{4}, 11 \lambda^{4}, 17 \lambda^{4}$ triboratribenzo[fgh,pqr,za $\left.\mathrm{b}_{1}\right]$ trinaphthylene (hereafter called $3 \mathrm{BNF}_{2}$ ), featuring triple boron (III)-cored chromophore with discotic triazatrinaphthylene as the heterocyclic ligand (Figure 1). ${ }^{[20]}$ This molecule can undergo cross-link both in solution and in the solid-state upon irradiation with UV and white light. In particular, we show for the first time that the $\mathrm{BF}_{2}$ groups exposed on such a family of dyes can reticulate when exposed to optical stimuli; such a finding is unusual since these groups attracted a great interest in view of their chemical and photo-stability. ${ }^{[21-22]}$ The photo-induced reaction can be achieved with common photolithography setups making this molecule interesting for applications as negative photoresist, e.g. for electronics. When blended with functional polymers, $3 \mathrm{BNF}_{2}$ can be cross-linked creating nodes that physically trap the other components transferring the crosslink properties to the blend without affecting the functional characteristics of the other 
components, making it possible to develop a new series of functional negative photoresist that, differently from what already reported in literature, ${ }^{[19]}$ can be directly written with commercial photolithography setups operating with standard $\lambda=405 \mathrm{~nm}$ laser.

As a result of the very low polarity of both the discotic aromatic core and of the alkyl sidechains, $3 \mathrm{BNF}_{2}$ is well soluble in non-polar solvents such as dichloromethane $\left(\mathrm{CH}_{2} \mathrm{Cl}_{2}\right)$ which is also a good solvent for many semiconducting synthetic polymers. Such a solvent was chosen because it is known to exhibit a greater stability to light irradiation compared to other chlorinated solvents like chloroform.

Figure 2 displays the evolution of the UV-Vis absorption and fluorescence spectra of $3 \mathrm{BNF}_{2}$ in $\mathrm{CH}_{2} \mathrm{Cl}_{2}$ solution, under exposure to white light (power density $=232.7 \mathrm{mWcm}^{-2}$ measured at $\lambda=405 \mathrm{~nm}$ ). Upon irradiation the UV-Vis spectra (Figure 2A, C) show a gradual decrease of the peaks at $\lambda=247,286$ and $368 \mathrm{~nm}$ and a small increase of absorbance around $\lambda=448$ and $313 \mathrm{~nm}$. The result of this absorption change is evidenced by the appearance of 3 isosbestic points at $\lambda=293,337$ and $428 \mathrm{~nm}$ and an overall loss in the fine structure of the spectra. The fluorescence spectra (Figure 2B, D) were recorded on the same solutions using an excitation wavelength of $\lambda=293 \mathrm{~nm}$; such an excitation wavelength, at the isosbestic point, was chosen to avoid the errors in emission intensity due to the variation of the solution's extinction coefficient, although the same results in fluorescence spectra were recorded using any excitation wavelength between $\lambda=240$ and $425 \mathrm{~nm}$. Upon light exposure the fluorescence spectra in Figure 2B, D reveal a gradual disappearance of the emission band at $\lambda=471 \mathrm{~nm}$ and the appearance of a more red-shifted band. Noteworthy, both the kinetic of the processes and the position of the new peak in fluorescence depend on the concentration of the irradiated solutions: for highly concentrated solutions such as $1 \times 10^{-4} \mathrm{M}$ (Figure $2 \mathrm{~A}, \mathrm{~B}$ ), the whole process requires an exposure of $420 \mathrm{~s}$ and the new fluorescence peak is pointed at $\lambda$ $=520 \mathrm{~nm}$ whereas for less concentrated solutions, such as $8 \times 10^{-6} \mathrm{M}$, the process is not 
completed yet even after $1690 \mathrm{~s}$ and the new fluorescence peak is observed at $\lambda=511 \mathrm{~nm}$. The faster kinetic and the larger red-shift at higher concentrations suggest the formation of aggregates upon irradiation. This can be confirmed by performing measurements of Resonance Light Scattering (RLS) after different steps of irradiation (Figure 3). RLS is a highly sensitive method to study the aggregation of chromophores such as porphyrins in solution using the scattered light close to the absorbance wavelength of the sample. ${ }^{[23]}$ Figure 3 displays an overlay between UV-vis absorbance and RLS of a $4.86 \times 10^{-5} \mathrm{M}$ solution in $\mathrm{CH}_{2} \mathrm{Cl}_{2}$ (optical path $=10 \mathrm{~mm}$ ) before and after light irradiation. It reveals that increasing exposure to white light leads to an increase of the resonance scattering peak around $\lambda=500$ $\mathrm{nm}$ which is accompanied by a red-shift. This behaviour is typical for aggregated chromophores with a weak exciton coupling. ${ }^{[24]}$ On the other hand, the disappearance of the peaks at ca. $\lambda=320 \mathrm{~nm}$ can be considered an artifact related to the increased extinction band that covers the signal at that wavelength.

A similar evolution of the UV-vis absorbance upon light irradiation can be observed also in thin films (Figure S1) but, in the case of solid films, the decrease of absorbance is more pronounced for the peak at $\lambda=340 \mathrm{~nm}$.

To gain insight into the role of the light and the reasons of the decreased absorbance, fresh solutions were prepared in deuterated $\mathrm{CH}_{2} \mathrm{Cl}_{2}\left(\mathrm{CD}_{2} \mathrm{Cl}_{2}\right)$ and analysed with NMR spectroscopy before and after light exposure. Figures S2, S3, S4 and S5 in the Supporting Information portray the ${ }^{1} \mathrm{H},{ }^{13} \mathrm{C},{ }^{19} \mathrm{~F}$ and ${ }^{11} \mathrm{~B}$ NMR before and after $120 \mathrm{sec}$ and $420 \mathrm{sec}$ of white light exposure. The resulting NMR spectra show no differences in the case of ${ }^{1} \mathrm{H},{ }^{13} \mathrm{C}$ and ${ }^{11} \mathrm{~B}$ and just a small shift to higher ppm values in the case of ${ }^{19} \mathrm{~F}$ after irradiation. Such a finding provides us two different information. On the one hand, it confirms that the molecule is not degraded during the irradiation process. On the other hand, it suggests that the photoinduced reaction takes place without modifying the chemical environment of the involved 
atoms. This reaction most likely involves an attack from the nitrogen of an aniline to the boron of an adjacent molecule, resulting in just an exchange of the coordinated boron of each aniline. Moreover, the presence of three $\mathrm{N}_{-} \mathrm{BF}_{2}$ groups per molecule ensure the formation of cross-linked networks. This model is in agreement with X-ray Photoelectron Spectroscopy (XPS) study on thin uniform films of $3 \mathrm{BNF}_{2}$ in which some areas were exposed to different doses of light irradiation using the UV $(\lambda=405 \mathrm{~nm})$ laser of a laser writer setup. The XPS spectra (Figure S6) reveal that the peaks of carbon $(\mathrm{C} 1 \mathrm{~s})$ and nitrogen $(\mathrm{N} 1 \mathrm{~s})$ are almost unchanged with increasing the irradiation dose whereas the peaks of boron (B1s) and fluorine (F1s) are shifted to higher binding energies. Such an observation strongly supports the hypothesis of the formation of new bonds that involves the $\mathrm{BF}_{2}$ groups that behave as intermolecular bridges, in good agreement with the UV-vis, fluorescence, RLS and NMR data previously discussed.

Taking advantage of these photo-induced aggregation properties, $3 \mathrm{BNF}_{2}$ was used as negative photoresist. The use of different deposition techniques makes it possible to vary the morphology of the obtained film: while spin-coating leads to the formation of uniform amorphous films, crystals can be formed by drop-casting (Figure S7) and fibers are obtained by using solvent induced precipitation (Figure S8). ${ }^{[25]}$ In all three cases cross-linking is obtained with UV irradiation, leading to insoluble and non meltable materials. This was evidenced by optical microscopy studies carried out by irradiating a sample of $3 \mathrm{BNF}_{2}$ fibers using the light source of a fluorescence microscope $(\lambda=450-500 \mathrm{~nm}$, power density $=3312.7$ $\mathrm{mW} \mathrm{cm}^{-2}$ ) for 5 minutes and by performing thermal annealing up to $200{ }^{\circ} \mathrm{C}$ (Figure S9 A) followed by further rinsing in $\mathrm{CH}_{2} \mathrm{Cl}_{2}$ (Figure S9 B). The images in Figure S9 reveals that the unexposed areas get melt with the annealing and removed by the solvent whereas the exposed ones exhibit morphologies that are unaltered after both steps. This observation is supported by Differential Scanning Calorimetry (DSC) measurements in Figure S14 showing a 
transition peak at $158^{\circ} \mathrm{C}$ that is not observed when repeating the measurement on the same sample after exposure to white light. ${ }^{1} \mathrm{H}-\mathrm{NMR}$ and the XPS analyses provide evidence that the cross-link reaction involves the $\mathrm{BF}_{2}$ groups that react among each other at the intermolecular level without affecting neither the molecule's aromatic core nor the alkyl side chains. This result implies that if the $3 \mathrm{BNF}_{2}$ is blended with a polymer in the solid state, the UV-irradiation activated cross-linking process can be used to trap the polymer to make it insoluble, keeping its structure and functional properties unchanged. To prove this hypothesis, we blended $3 \mathrm{BNF}_{2}$ with polystyrene $(\mathrm{PS}$, molecular weight $=500 \mathrm{kDa})$ by preparing $3 \mathrm{mg} \mathrm{ml}^{-}$ ${ }^{1}$ solution in $\mathrm{CH}_{2} \mathrm{Cl}_{2}$ with a content of $7 \%$ in weight of $3 \mathrm{BNF}_{2}$. The solutions were deposited by spin-coating on $\mathrm{Si} / \mathrm{SiO}_{2}$ to generate uniform amorphous films, and irradiated with the light source of a fluorescence microscope. Figure $\mathrm{S} 10$ shows that, after rinsing the film in $\mathrm{CH}_{2} \mathrm{Cl}_{2}$, the unexposed areas are completely removed while the exposed ones are unchanged as a result of the interaction with the solvent. To test the applicability of the PS- $3 \mathrm{BNF}_{2}$ blend as photoresist, a commercial laser writer setup, equipped with a $405 \mathrm{~nm}$ laser source for maskless lithography, was used. As first step the sample's surface was covered with markers to identify up to 81 square areas of $250 \times 250 \mu \mathrm{m}^{2}$. These squares were then written with the 405 $\mathrm{nm}$ laser with different doses to find the threshold and the optimal doses to cross-link the film (Figure S11). The explored range of doses ranges from 13 to $11061 \mathrm{~mJ} \mathrm{~cm}^{-2}$; the effect of multiple repetition (up to 10) was also explored, reaching a maximum total dose of $100 \mathrm{~J} \mathrm{~cm}^{-2}$ exposing the same area 10 times with a $10000 \mathrm{~mJ} \mathrm{~cm}^{-2}$ beam. By using the optical microscopy in black and white mode it is possible to roughly estimate the best exposure conditions measuring the difference in optical contrast between the cross-linked film and the clean substrate after rinsing the sample. Figure S12 shows that the minimum dose to achieve the cross-link is ca. $2100 \mathrm{~mJ} \mathrm{~cm}^{-2}$; above $5500 \mathrm{~mJ} \mathrm{~cm}^{-2}$ for 10 repetitions a plateau is reached rendering unnecessary the use of higher energies. In order to estimate the maximum 
resolution that can be achieved with this system, the same laser writer setup was used to write more complex patterns. One example is reported in Figure 4 in which the "Nanochemistry" logo was written on a PS-3BNF 2 film by using a dose of $5500 \mathrm{~mJ} \mathrm{~cm}^{-2}$ (10 repetitions). The fluorescence microscopy image in the inset (figure 4C), acquired after the film development, displays well-resolved characters even for spacing smaller than $2 \mu \mathrm{m}$.

Another step forward in the development of functional photoresists is the use of electroactive polymers replacing PS in blend. As a proof-of-concept we decided to use a semiconducting polymer, i.e. the Poly $\{[\mathrm{N}, \mathrm{N} 9-b i s(2-o c t y l d o d e c y l)-n a p h t h a l e n e-1,4,5,8$-bis(dicarboximide)2,6-diyl]-alt-5,59-(2,29-bithiophene)\}, P(NDI2OD-T2), Polyera ActivInk N2200. The $\mathrm{P}(\mathrm{NDI} 2 \mathrm{OD}-\mathrm{T} 2)$ is an n-type polymer combining high charge carrier mobilities and good air stability. ${ }^{[26]}$ Since the 3BNF2 alone behaves as an electrical insulator, the combination of the latter with P(NDI2OD-T2) appears particularly suitable for these experiments. Moreover the absorption spectrum of P(NDI2OD-T2) shows a minimum between $\lambda=450$ and $550 \mathrm{~nm},{ }^{[27]}$ making it compatible with the absorbance of the $3 \mathrm{BNF}_{2}$ and with the light source of the fluorescence microscope used for the preliminary tests. By varying systematically the content of $3 \mathrm{BNF}_{2}$ in the blend with $\mathrm{P}(\mathrm{NDI} 2 \mathrm{OD}-\mathrm{T} 2)$ to make writable operating electrical devices such as field-effect transistors (FETs) we found $20 \%$ in a $3 \mathrm{mg} \mathrm{ml}^{-1}$ solution of P(NDI2ODT2) in $\mathrm{CH}_{2} \mathrm{Cl}_{2}$ being the optimal compromise between electrical characteristic and amount of cross-link centers. The panel in Figure 5 portrays the transfer and output characteristics of bottom-gate bottom-contact FETs made by pure P(NDI2OD-T2) (Figure 5A, B), blend of $\mathrm{P}(\mathrm{NDI} 2 \mathrm{OD}-\mathrm{T} 2) / 3 \mathrm{BNF}_{2}($ Figure $5 \mathrm{C}, \mathrm{D})$ and blend of $\mathrm{P}(\mathrm{NDI} 2 \mathrm{OD}-\mathrm{T} 2) / 3 \mathrm{BNF}_{2}$ after exposure to the light of the fluorescence microscope (power density $=3312.7 \mathrm{~mW} \mathrm{~cm}^{-2}$ for 20 minutes) and rinsing in $\mathrm{CH}_{2} \mathrm{Cl}_{2}$ (Figure 5E, F). The extracted field-effect mobility of these devices amount to $8.6 \times 10^{-3} \mathrm{~cm}^{2} \mathrm{~V}^{-1} \mathrm{~s}^{-1}$ for the pristine sample, $2.4 \times 10^{-3} \mathrm{~cm}^{2} \mathrm{~V}^{-1} \mathrm{~s}^{-1}$ for the blend before exposure, and $5.0 \times 10^{-5} \mathrm{~cm}^{2} \mathrm{~V}^{-1} \mathrm{~s}^{-1}$ for the blend after exposure and rinsing. The presence of the 
$20 \%$ of $3 \mathrm{BNF}_{2}$ inside the blend is accompanied by a decrease in mobility by a factor of 4 compared to the blank made with pure P(NDI2OD-T2). Such an effect can be ascribed to either or both the morphology or energetic changes since small molecules in blend with semiconducting polymers can act as scattering centers for the charge carriers. ${ }^{[28]}$ Nevertheless, $3 \mathrm{BNF}_{2}$ has neither energetic interaction nor it behaves as trap for charge carriers of $\mathrm{P}(\mathrm{NDI} 2 \mathrm{OD}-\mathrm{T} 2)$ since its energetic levels falls outside the band-gap of the semiconducting polymer. In fact, the HOMO and LUMO levels of P(NDI2OD-T2) are positioned at $-5.36 \mathrm{eV}$ and $-3.91 \mathrm{eV},{ }^{[29]}$ respectively, whereas the HOMO and LUMO of $3 \mathrm{BNF}_{2}$ as determined by Cyclic Voltammetry $(\mathrm{CV})$ and optical bandgap measurements amount to $-5.97 \mathrm{eV}$ and $-3.17 \mathrm{eV}$, respectively.

After rinsing the sample, the extracted mobility of the devices drops down by a factor of 50 . It is however fair to note that during the whole treatment the samples were exposed to air for several hours during the irradiation steps, thus partial sample oxidation may have occurred. Furthermore, as displayed in Figure S13 the device's area is bigger than the microscope's spot; as a result, after the development, some parts of the device get uncovered, thereby decreasing the overall effective coverage. The extracted average mobility, corrected in light of the effective coverage, amounts to $9.5 \times 10^{-5} \mathrm{~cm}^{2} \mathrm{~V}^{-1} \mathrm{~s}^{-1}$. Albeit the field-effect mobilities recorded are far from state-of-the-art, they provide clear evidence for the versatility of potential of our patterning method for functional application, e.g. in electronics, with the additional bonus of allowing unconventional patterns to be simply tailored.

In summary, we have shown for the first time that boron-cored chromophore derivatives can be cross-linked via exposure to white and UV light in solution and in solid state by using commercial photolithography setups, thereby be exploited as negative photoresist. The photoinduced reaction involves uniquely the bonding of $\mathrm{BF}_{2}$ groups belonging to adjacent molecules, leaving the other molecular functions unaffected. We showed that these molecules 
can act as reticulation centers to transfer the photoresist properties to functional polymers thereby developing functional photoresist by simply blending the components. This approach is extremely versatile and can be applied to a wide range of functional polymers for different technological applications to directly write functional patterns or layer-by-layer depositions for multicomponent devices using cycles of depositions and light exposure.

\section{Experimental Section}

Detailed experimental data can be found in supporting information.

\section{Supporting Information}

Supporting Information is available from the Wiley Online Library or from the author.

\section{Acknowledgements}

The authors thank Dr. Giovanna De Luca for enlightening discussions on the RLS results. This work was financially supported by the EC through the European Research Council project SUPRAFUNCTION (GA-257305), the Marie Sklodowska-Curie ITN projects SYNCHRONICS (GA 643238) and iSwitch (GA No. 642196), the FP7-NMP-2012-SMALL6 "SACS" project (GA-310651), the Agence Nationale de la Recherche through the LabEx project Chemistry of Complex Systems (ANR-10-LABX-0026_CSC), and the International Center for Frontier Research in Chemistry (icFRC).

Received: ((will be filled in by the editorial staff))

Revised: ((will be filled in by the editorial staff)) Published online: ((will be filled in by the editorial staff))

\section{References}


[1] A. C. Arias, J. D. MacKenzie, I. McCulloch, J. Rivnay, A. Salleo, Chem. Rev. 2010, 110, 3-24.

[2] M. A. C. Stuart, W. T. S. Huck, J. Genzer, M. Mueller, C. Ober, M. Stamm, G. B. Sukhorukov, I. Szleifer, V. V. Tsukruk, M. Urban, F. Winnik, S. Zauscher, I. Luzinov, S. Minko, Nat. Mater. 2010, 9, 101-113.

[3] C. D. Dimitrakopoulos, P. R. L. Malenfant, Adv. Mater. 2002, 14, 99.

[4] H. Sirringhaus, Adv. Mater. 2005, 17, 2411-2425.

[5] M. Mas-Torrent, C. Rovira, Chem. Soc. Rev. 2008, 37, 827-838.

[6] R. H. Friend, R. W. Gymer, A. B. Holmes, J. H. Burroughes, R. N. Marks, C. Taliani, D. D. C. Bradley, D. A. Dos Santos, J. L. Bredas, M. Logdlund, W. R. Salaneck, Nature 1999, 397, 121-128.

[7] C. J. Brabec, S. Gowrisanker, J. J. M. Halls, D. Laird, S. Jia, S. P. Williams, $A d v$. Mater. 2010, 22, 3839-3856.

[8] S. W. Thomas, G. D. Joly, T. M. Swager, Chem. Rev. 2007, 107, 1339-1386.

[9] J. A. DeFranco, B. S. Schmidt, M. Lipson, G. G. Malliaras, Org. Electron. 2006, 7, 22-28.

[10] M. L. Chabinyc, A. Salleo, Chem. Mater. 2004, 16, 4509-4521.

[11] H. Sirringhaus, T. Kawase, R. H. Friend, T. Shimoda, M. Inbasekaran, W. Wu, E. P. Woo, Science 2000, 290, 2123-2126.

[12] M. Baklar, P. H. Wobkenberg, D. Sparrowe, M. Goncalves, I. McCulloch, M. Heeney, T. Anthopoulos, N. Stingelin, J. Mater. Chem. 2010, 20, 1927-1931.

[13] S. Nau, C. Wolf, K. Popovic, A. Blumel, F. Santoni, A. Gagliardi, A. di Carlo, S. Sax, E. J. W. List-Kratochvil, Adv. Electron. Mater. 2015, 1.

[14] B. Bao, J. Jiang, F. Li, P. Zhang, S. Chen, Q. Yang, S. Wang, B. Su, L. Jiang, Y. Song, Adv. Funct. Mater. 2015, 25, 3286-3294.

[15] L. J. Guo, Adv. Mater. 2007, 19, 495-513.

[16] M. Cavallini, C. Albonetti, F. Biscarini, Adv. Mater. 2009, 21, 1043-1053.

[17] M. X. Kuang, L. B. Wang, Y. L. Song, Adv. Mater. 2014, 26, 6950-6958.

[18] K. J. Byeon, H. Lee, Eur. Phys. J. Appl. Phys 2012, 59, 21.

[19] R. Q. Png, P. J. Chia, J. C. Tang, B. Liu, S. Sivaramakrishnan, M. Zhou, S. H. Khong, H. S. O. Chan, J. H. Burroughes, L. L. Chua, R. H. Friend, P. K. H. Ho, Nat. Mater. 2010, 9, 152-158.

[20] V. Treibs, Alfred, F. H. Kreuzer, Justus Liebigs Ann. Chem. 1969, 718, 105-115.

[21] A. Loudet, K. Burgess, Chem. Rev. 2007, 107, 4891-4932.

[22] G. Ulrich, R. Ziessel, A. Harriman, Angew. Chem. Int. Ed. 2008, 47, 1184-1201.

[23] R. F. Pasternack, P. J. Collings, Science 1995, 269, 935-939.

[24] R. F. Pasternack, C. Bustamante, P. J. Collings, A. Giannetto, E. J. Gibbs, J. Am. Chem. Soc. 1993, 115, 5393-5399.

[25] D. H. Kim, D. Y. Lee, H. S. Lee, W. H. Lee, Y. H. Kim, J. I. Han, K. Cho, Adv. Mater. 2007, 19, 678 .

[26] H. Yan, Z. Chen, Y. Zheng, C. Newman, J. R. Quinn, F. Dotz, M. Kastler, A. Facchetti, Nature 2009, 457, 679-686.

[27] R. Steyrleuthner, M. Schubert, I. Howard, B. Klaumunzer, K. Schilling, Z. Chen, P. Saalfrank, F. Laquai, A. Facchetti, D. Neher, J. Am. Chem. Soc. 2012, 134, 1830318317.

[28] E. Orgiu, N. Crivillers, M. Herder, L. Grubert, M. Paetzel, J. Frisch, E. Pavlica, D. T. Duong, G. Bratina, A. Salleo, N. Koch, S. Hecht, P. Samorì, Nat. Chem. 2012, 4, 675679.

[29] Z. Chen, Y. Zheng, H. Yan, A. Facchetti, J. Am. Chem. Soc. 2009, 131, 8-9. 


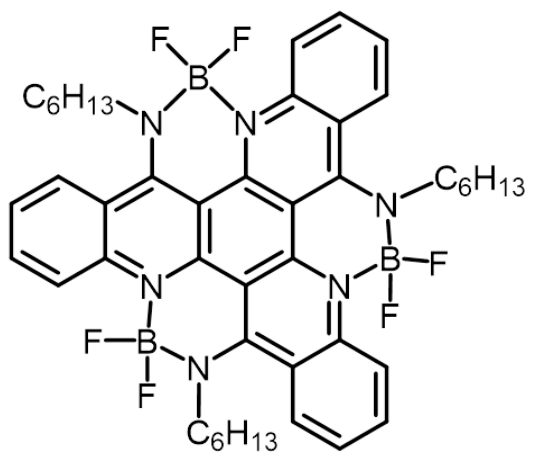

Figure 1. Molecular structure of $3 \mathrm{BNF}_{2}$.
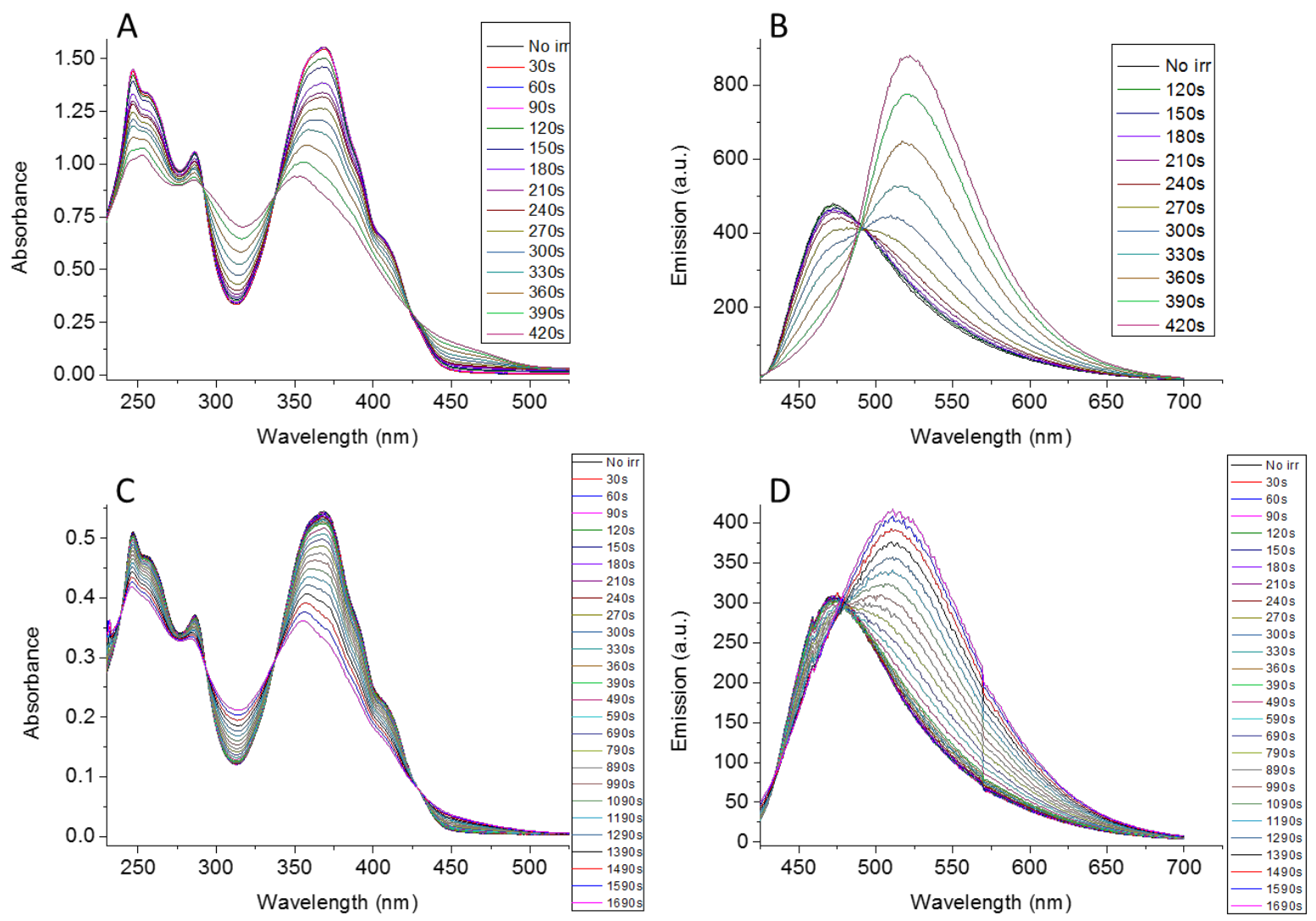

Figure 2. Optical properties of $3 \mathrm{BNF}_{2}$ in $\mathrm{CH}_{2} \mathrm{Cl}_{2}$ solution during white light irradiation. (A, C) UV-Vis absorption and (B, D) fluorescence spectra. $(A, B)$ Concentration $=1 \times 10^{-4} \mathrm{M} ;(\mathrm{C}, \mathrm{D})$ Concentration $=8 \times 10^{-6} \mathrm{M}$. (B,D) Excitation at $\lambda=293 \mathrm{~nm}$. 


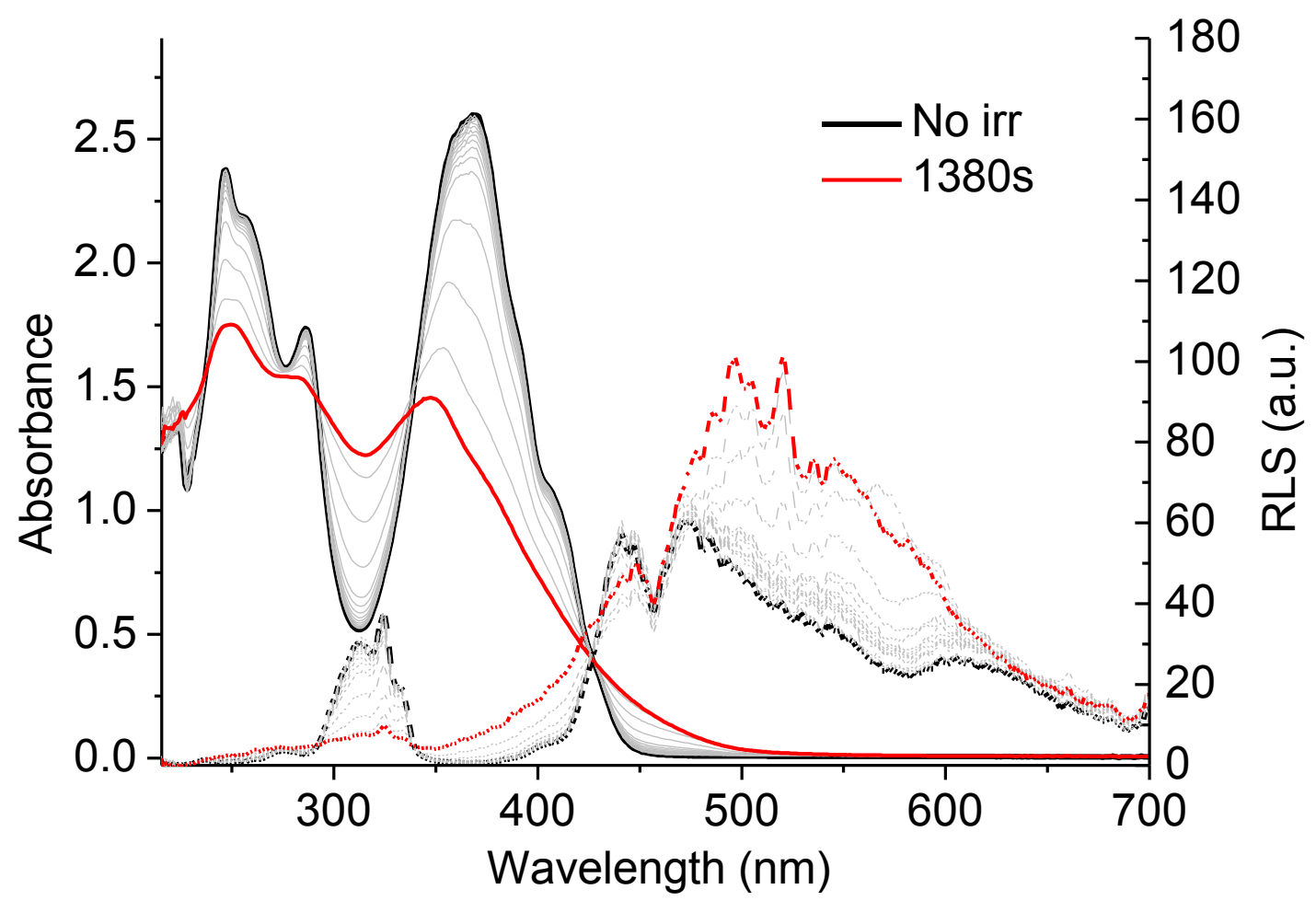

Figure 3. Overlay between UV-absorbance and RLS spectra of $3 \mathrm{BNF}_{2}$ before and after $1380 \mathrm{~s}$ of light irradiation $\left(\mathrm{C}=4.87 \times 10^{-5} \mathrm{M}\right.$ in $\left.\mathrm{CH}_{2} \mathrm{Cl}_{2}\right)$.

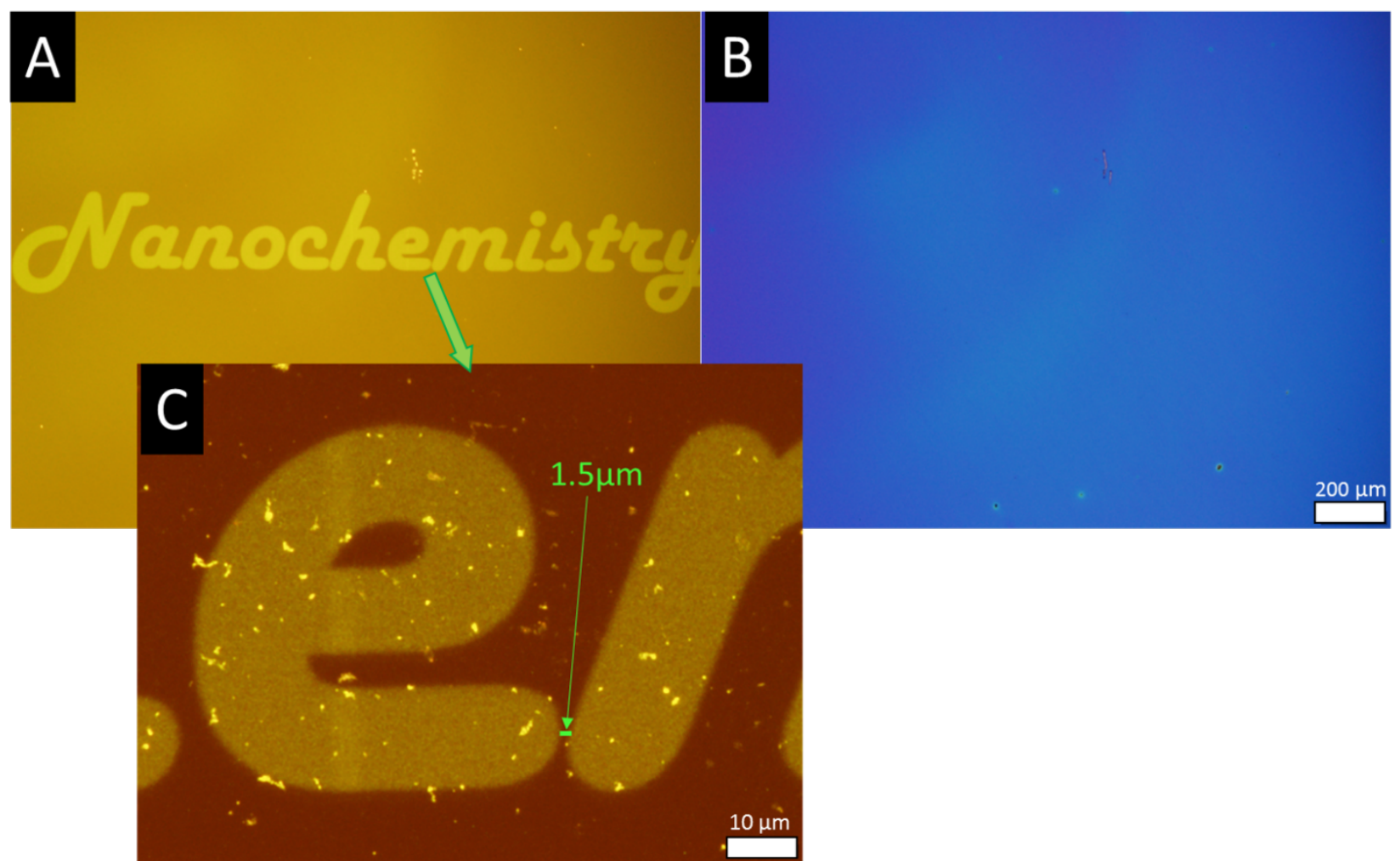

Figure 4. Optical and Fluorescence image on the Nanochemistry logo made by $3 \mathrm{BNF}_{2}-\mathrm{PS}$ on $\mathrm{SiO}_{2} / \mathrm{Si}_{(\mathrm{SiO}}$ thickness $=230 \mathrm{~nm})$. (A,B) show the sample after patterning in fluorescence and in bright field whereas (C) 
displays the fluorescence image after the development in $\mathrm{CH}_{2} \mathrm{Cl}_{2}$. The bright spots in the latter are residual from the development process.

A
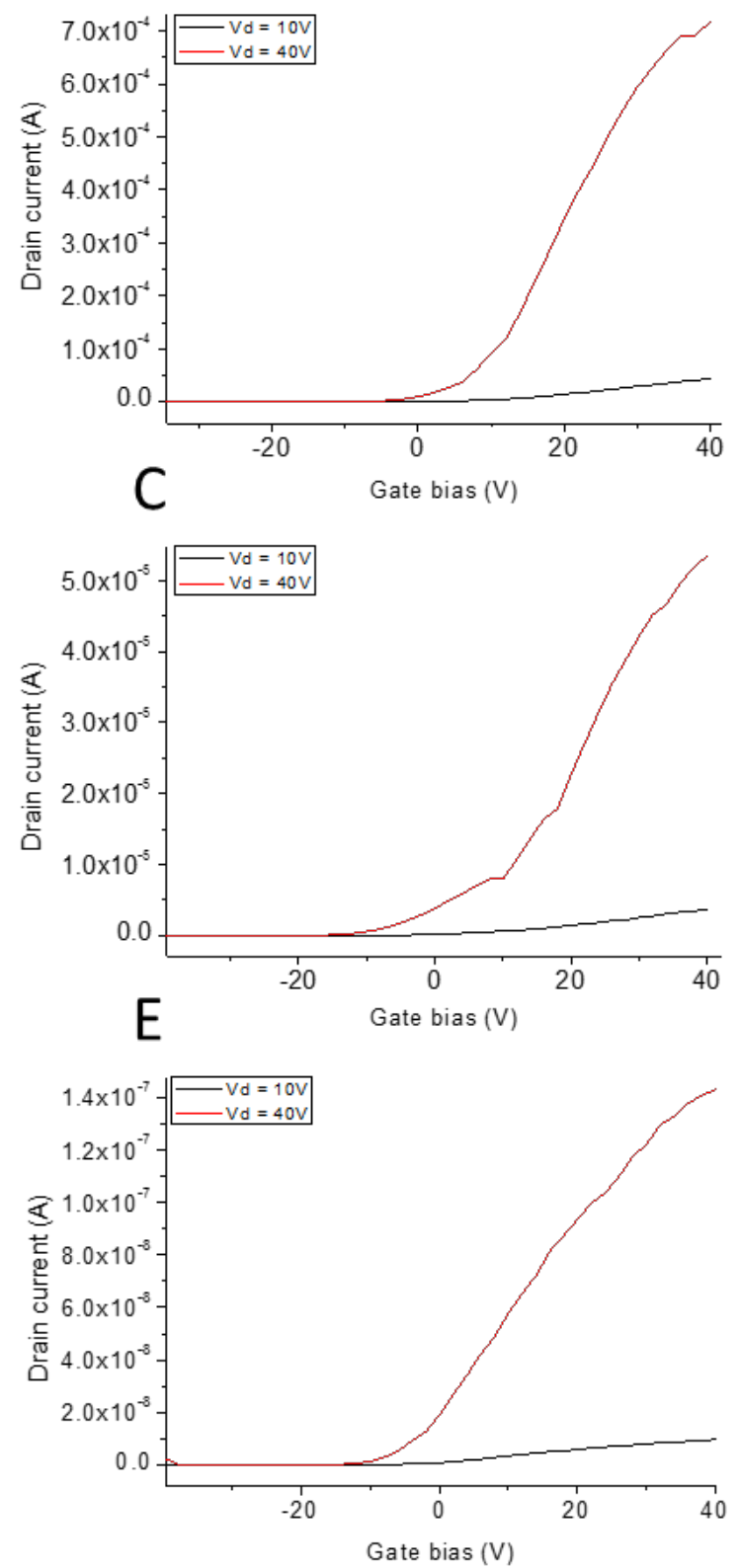

B
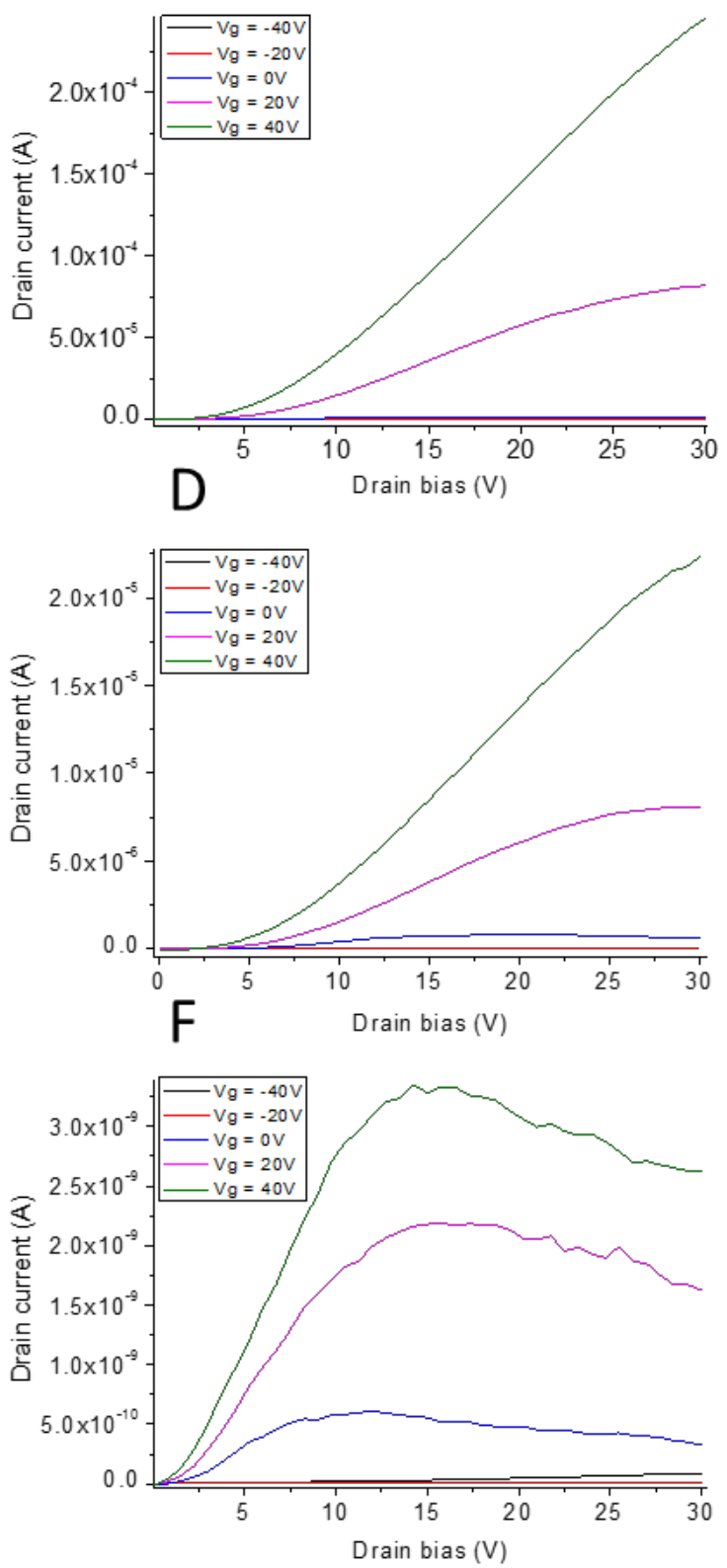

Figure 5. Electrical (A,C,E) transfer and $(B, D, F)$ output characteristic of $(A, B)$ pure $P(N D I 2 O D-T 2)$ reference, $(\mathrm{C}, \mathrm{D}) 3 \mathrm{BNF}_{2}-\mathrm{P}(\mathrm{NDI} 2 \mathrm{OD}-\mathrm{T} 2)$ blend before development, $(\mathrm{E}, \mathrm{F}) 3 \mathrm{BNF}_{2}-\mathrm{P}(\mathrm{NDI} 2 \mathrm{OD}-\mathrm{T} 2)$ blend after development. 